\title{
Compact Electrothermal Modeling of an X-band MMIC
}

\author{
Sonali Luniya* William Batty ${ }^{\dagger}$ Vincent Caccamesi ${ }^{\ddagger}$ Mikael Garcia $^{\ddagger}$ Carlos Christoffersen $^{\S}$ \\ Samson Melamed* W. Rhett Davis* and Michael Steer* \\ * Department of Electrical and Computer Engineering, North Carolina State University, Raleigh, NC \\ $\dagger$ Filtronic Compound Semiconductors Ltd., Newton Aycliffe, UK, \\ $\ddagger$ Raytheon, Sudbury, MA \\ $\S$ Department of Electrical and Computer Engineering, Lakehead University, Thunder Bay, Ontario, CA
}

\begin{abstract}
Compact electrothermal modeling of lumped electrical devices and compact thermal modeling of volumetric materials enables efficient electrothermal modeling of microwave circuits. The compact thermal model of the body of an X-band MMIC is based on analytical solutions of the heat diffusion equation in thermal sub-volumes. The model is accurate and captures thermal nonlinearities. The model considers complex MMIC features such as surface metallization and vias, as well as the mounting configurations including lead-frame, carrier, and printed circuit board. This is coupled with electrothermal models of transistors and of resistors. The models are incorporated in a multi-physics simulator that uses the same model in both transient and harmonic analysis of an X-band LNA MMIC. Simulations are validated with steady-state thermal measurements.
\end{abstract}

Index Terms-MMIC, electrothermal effects, circuit simulation, modeling.

\section{INTRODUCTION}

Co-simulation of the thermal and electrical characteristics of active microwave circuits is notoriously slow with important electrical transients being in the picosecond range while package-level thermal transients can be a second or longer. In the region close to heat generating devices such as transistors and resistors the thermal transients are taken as milliseconds and simulation schemes have evolved that simulate the electrical circuit at picosecond or smaller time-steps while updating the thermal environment every millisecond or so. However, in the active device region, the heat generating mechanisms are at the electrical signal rate. In the micron and submicron longitudinal dimensions of the active channels of transistors the thermal environment is changing at the rate of the electrical signal. There can be tight coupling of the temperature and active device performance, for example through temperature dependent mobility. While this effect can be viewed as relatively small the effect on performance can be significant in terms of some levels of performance such as intermodulation distortion with a two-tone signal or adjacent channel distortion with digitally-modulated signals. In the latter case distortion that is $-80 \mathrm{dBc}$ is important, this is 1 part in 10,000 in terms of voltage levels. So even very low levels of distortion are important and the electrothermal interactions must be captured at the carrier period rate or at least at the envelope rate.

Thermal simulations are traditionally performed using Finite Element Modeling (FEM) of 3D structures. These are computationally intensive as volumetric discretization is involved. It is computationally prohibitive to use FEM thermal simulations in conjunction with electrical simulations. As a result considerable effort has been expended in developing compact thermal models that can be computed many of orders of magnitude faster than can FEM schemes. The simplest thermal model is to use simple arrangements of thermal resistances and capacitances together with a circuit-based thermal source, typically a controlled voltage or current source, whose amplitude is proportional to the power dissipated in electrical devices. Electrothermal circuit simulation has been performed previously utilising compact thermal models based on simplified package structures. These often represented multifinger devices by only a single, average heat dissipating area and ignored all device fine structure such as die surface metallisation. Examples are APLAC, with a simple thermal description by Veijola et al. [4], based on heat dissipating spheres; simulation of IGBTs in circuit simulator SABER [5], utilising the approximate thermal model of Hefner and Blackburn for die, carriers and heatsinks; and SISSI by V. Szekely et al. [6], based on a thermal RC-ladder network obtained from simulated or measured time constant spectra. Various thermal resistance calculation techniques using Green's functions, Fourier series, conformal mapping, extraction using deconvolution from numerically generated and measured thermal responses, and numerical techniques have be applied to individual circuit components, devices, heat sinks and packages.

In this paper we describe a compact thermal model of a MMIC body. The model is in effect an 'interface element' equivalent of a volumetric model. This goes beyond the typical boundary-element formulation, which discretises whole surfaces, and discretises only interfaces between subvolumes. Transformations enable the non linear volume to be described by an analytical thermal impedance matrix model while still capturing full thermal capacitance. The thermal impedance matrix is reduced to capture the interactions from one point on the discretized interface 
to another. This allows description of complex MMIC features such as surface metallization and via holes, and package and mounting configurations, such as epoxy or solder attach to lead-frame paddle or carrier. It is critical to realize that the compact thermal model captures nonlinearthermal effects as well as thermal capacity effects. The electro-thermal model of the MMIC is based on the Curtice Cubic model with temperature coefficient parameters that capture the effect of temperature on mobility and on threshold voltage. The thermal terminals of the electrothermal GaAs pHEMT model are connected to the thermal model of the MMIC body. The result is un-compromised electro-thermal co-simulation of an X-band LNA MMIC. Results are in part verified using calibrated thermal images of a MMIC taken with an infra-red camera.

\section{Global State Variable based Modeling}

The electrothermal modeling presented in this paper is implemented in a global state variable based multidomain simulator, fREEDA ${ }^{\mathrm{TM}}$ (www.freeda.org). This simulator uses state variables to model various nonlinear devices. The state variable based parameterized nonlinear devices can be described with the following set of equations:

$$
\begin{aligned}
& \mathbf{v}_{N L}(t)=u\left[\mathbf{x}(t), \frac{d \mathbf{x}}{d t}, \ldots, \frac{d^{m} \mathbf{x}}{d t^{m}}, \mathbf{x}_{D}(t)\right] \\
& \mathbf{i}_{N L}(t)=w\left[\mathbf{x}(t), \frac{d \mathbf{x}}{d t}, \ldots, \frac{d^{m} \mathbf{x}}{d t^{m}}, \mathbf{x}_{D}(t)\right]
\end{aligned}
$$

where $\mathbf{v}_{N L}(t), \mathbf{i}_{N L}(t)$ are vectors of voltages and currents at the common ports, $\mathbf{x}(t)$ is a vector of state variables and $\mathbf{x}_{D}(t)$ a vector of time-delayed state variables, i.e., $\mathbf{x}_{D i}(t)=x_{i}\left(t-\tau_{i}\right)$. The time delays $\tau_{i}$ may be functions of the state variables. All the vectors in Eq. (1) and Eq. (2) have a same size $n_{d}$ equal to the number of common (device) ports. The general formulation to solve the system of linear and nonlinear devices is described in [1]. To incorporate the thermal effects into the circuit simulator, the thermal model is made to look like an electrical circuit. The thermal and electrical circuits are then solved simultaneously as if they were one large electrical circuit. The concept of local reference groups [2] helps to integrate the thermal network and guarantees no mixing of the electric and thermal currents. A general integration of an electrothermal network is shown in Fig. 1. Power dissipations in the active devices are represented as heat current sources referenced to thermal ground. The thermal ground is taken as $0 \mathrm{~K}$, and is treated in the circuit simulator as a local reference node. Thus, the entire thermal network is treated by the circuit simulator as a single local reference group. At the interface between the thermal component and thermal network are temperature, $\mathbf{T}$ 's, and heat flow, P's, variables. The error function at the thermal network interface is $\mathrm{P}=0$, which is equivalent to the error function of $\mathrm{I}=0$ in the electrical network. The

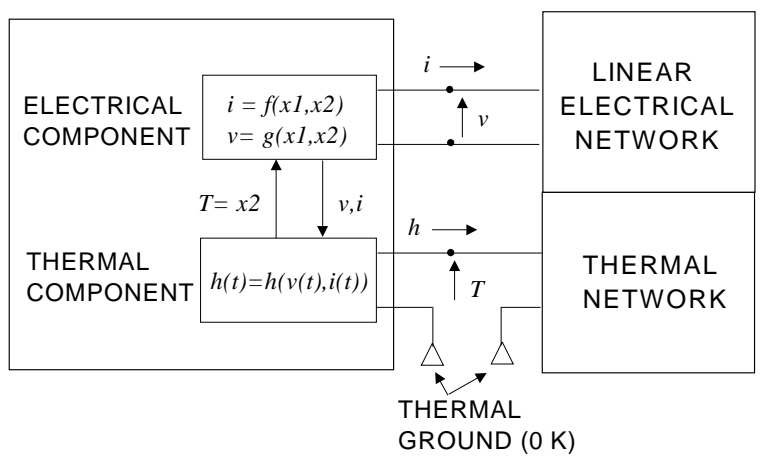

Fig. 1. An electrothermal element connected to linear and thermal networks.

temperature rise of the thermal network can be described by the equation:

$$
\triangle \theta_{\mathbf{i}}=\sum R_{\mathbf{T H}_{\mathbf{i j}}}(\mathbf{s}) P_{\mathbf{j}}
$$

where $\triangle \theta_{\mathbf{i}}$ is the Laplace transformed temperature rise of the element $i$ above its initial temperature, $R_{\mathbf{T H}_{\mathbf{i j}}}$ is the thermal impedance matrix in the Laplace s-space and the $P_{\mathrm{j}}$ are the transformed time-dependent fluxes due to power dissipation devices $j=1,2, . . i . . M$. The power dissipated by the nonlinear devices i.e. the heat currents into the thermal model are calculated by self-consistent electrical device models. These heat currents are picked as state variables of the thermal model. The thermal impedance network is calculated using the thermal impedance matrix model described in the next section.

\section{Thermal Impedance Matrix Model}

The construction of the thermal impedance matrix model, and its partial implementation in REEDA $^{\text {TM }}$, has been described at length in [3]. The model solves the non linear, time dependent heat diffusion equation in complicated thermal systems, by transformation of the equation to linear form, domain decomposition, and analytical solution of the transformed equation in regular subvolumes in complex frequency space, $s$.

The subvolume solutions take the form of $s$-space thermal impedance matrices, relating the transformed temperature rises of the heating elements and discretised interface elements, to corresponding power dissipations, as described in Eq. (3). The non linear, volumetric thermal problem is thus reduced to the discretised interfaces between subvolumes and the interface with the already non linear electrical model. Linear subsystems only have to be solved once, by thermal precomputation.

Explicit forms for the thermal impedance matrices are given in [3] and are of the general form,

$$
\begin{aligned}
R_{\mathbf{T H}_{\mathbf{i j}}}(\mathbf{s})=\sum_{m n}\left\{\begin{array}{c}
\operatorname{coth}\left(\gamma_{m n} D\right) \\
\operatorname{cosech}\left(\gamma_{m n} D\right)
\end{array}\right\} \times \\
\qquad \frac{-4 /\left(\kappa L W \gamma_{m n}\right)}{\left(1+\delta_{m 0}\right)\left(1+\delta_{n 0}\right)} \frac{I_{m n}^{i}}{I_{00}^{i}} \frac{I_{m n}^{j}}{I_{00}^{j}},
\end{aligned}
$$




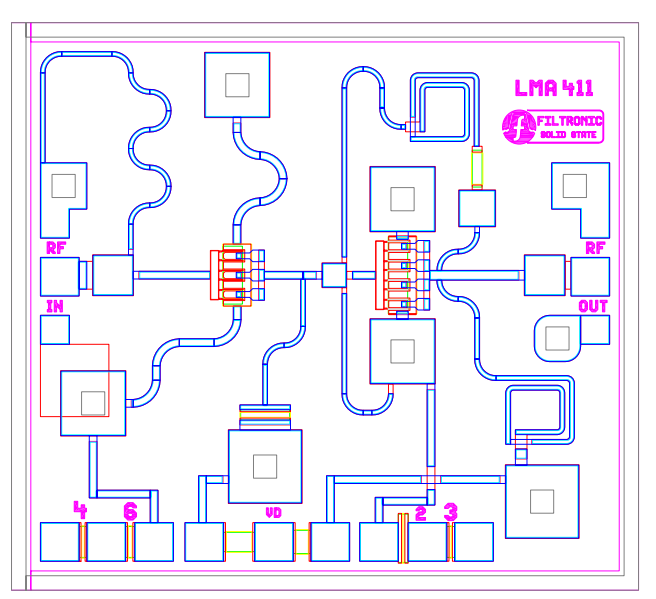

Fig. 2. Layout of LMA411 X-band MMIC.

where $m, n=0,1,2, \ldots$,

$$
\lambda_{m}=\frac{m \pi}{L}, \mu_{n}=\frac{n \pi}{W}, \gamma_{m n}=\left(\lambda_{m}^{2}+\mu_{n}^{2}+\frac{s}{k}\right)^{1 / 2},
$$

$L, W, D$ are the subvolume dimensions in the $x-, y-$ and $z$-directions respectively; the subvolume has thermal conductivity, $\kappa$, diffusivity, $k$; and $I_{m n}^{i}$ are area integrals over heating element and discretised interface element domains, $D_{i}$,

$$
I_{m n}^{i}=\iint_{D_{i}} \cos \lambda_{m} x \cos \mu_{n} y d x d y
$$

The $s$-space thermal impedance matrices are matrices of transfer functions representing the impulse responses of the thermal subsystems. They can be used directly in frequency space, by the evaluation $s \rightarrow j \omega$, to generate network parameters, for instance in harmonic balance simulation, or they can employ numerical Laplace inversion of $R_{T H_{i j}}(s) \frac{1}{s}$, to give system step response, for instance in convolution transient simulation.

In the simulations described here, the time-dependent thermal impedance matrices for thermal subsystems were generated in $f$ REEDA ${ }^{\mathrm{TM}}$. These thermal impedance matrices described thermal N-ports corresponding to subvolumes of the modelled MMIC. These matrices were used in the purely thermal transient simulations performed on the MMIC superstructure in $f$ REEDA ${ }^{\mathrm{TM}}$. Combination and reduction of N-ports, to form a global thermal impedance matrix describing the composite structure, was achieved by matrix manipulation on subsystem thermal impedance matrices. This was implemented in $f$ REEDA ${ }^{\mathrm{TM}}$ in terms of thermal conductances, allowing self-consistent electrothermal co-simulation with a harmonic balance analysis.

\section{Measurements And Results}

The electrothermal model described in the above sections was verified with thermal images of a high dynamic range Filtronics X-band MMIC LNA, shown in Fig. 2. This MMIC was glued with a thermally conductive epoxy to a Kovar substrate, which is screwed onto a carrier

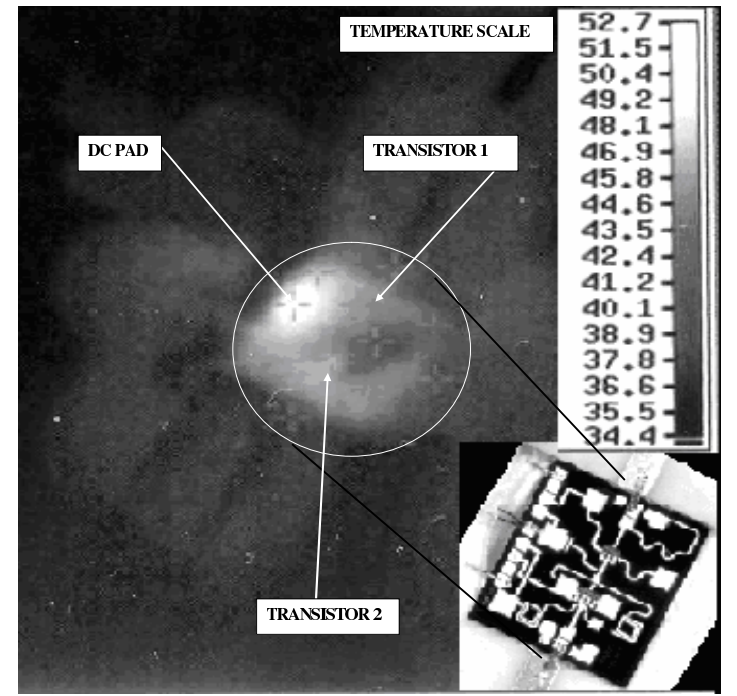

Fig. 3. Thermal image of the X-band MMIC.

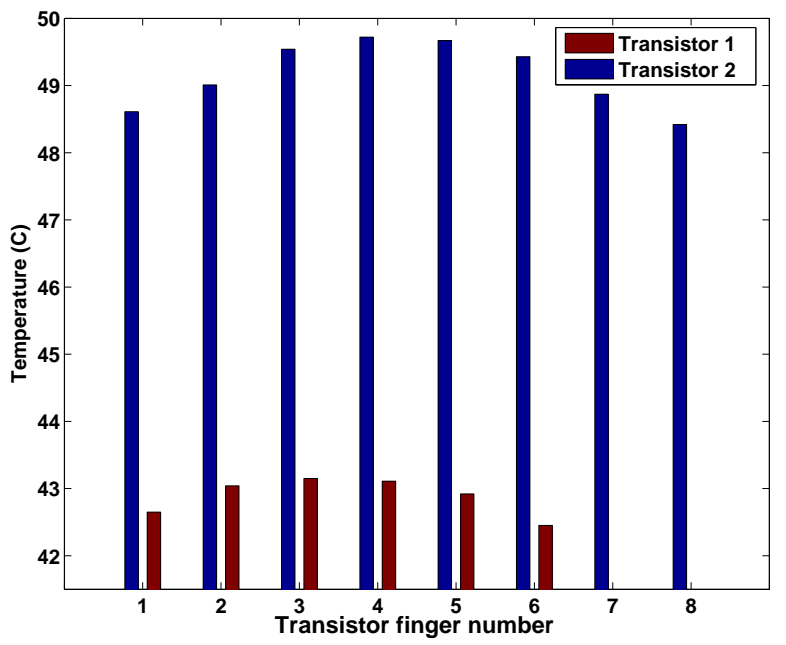

Fig. 4. Simulated temperatures of transistor fingers.

block, acting as the heat sink. The thermal images of the MMIC were captured using the infra red AFEMA Thermovision 900 SW/TE camera with a pixel size of $50 \mu \mathrm{m}$. The MMIC was painted with a proprietary black ink of known emissivity.

The GaAs die was modeled as an N-port with surface heating elements representing the 6 gates of transistor 1,8 gates of transistor 2 , and 2 power dissipating TaN resistors. In addition, 3 'pixel' elements were constructed, receiving zero power but delivering temperature response over $50 \mu \mathrm{m} \times 50 \mu \mathrm{m}$ 'pixel' areas, to mimic the resolution limitations of the thermal camera. The GaAs die N-port

TABLE I

TEMPERATURES $\left({ }^{\circ} \mathrm{C}\right)$ AT VARIOUS SPOTS ON THE MMIC.

\begin{tabular}{|c|c|c|c|}
\hline SPOT & $\begin{array}{c}\text { Simulated } \\
\text { Purely Thermal }\end{array}$ & $\begin{array}{c}\text { Simulated } \\
\text { Electrothermal }\end{array}$ & Measured \\
\hline \hline DC PAD & 46.9 & 46.1 & 46.4 \\
Transistor 1 & 41.3 & 40.5 & 40.7 \\
Transistor 2 & 44.7 & 43.3 & 43.1 \\
\hline
\end{tabular}


also contained surface discretisation for connection of surface metallisation. Metal elements were included to describe vias and via caps in the vicinity of transistor 2. These metal elements were in turn represented as thermal N-ports with discretised bases, and interface nodes connected to the corresponding discretised areas on the surface of the GaAs. The N-port reduction with matrix manipulation of subsystem thermal impedances, gave rise to a $19 \times 19$ global thermal impedance matrix, used in the electrothermal harmonic balance co-simulation.

The base of the GaAs die was also discretised, as were the top and bottom surfaces of the epoxy subvolume, and the top of the Kovar layer. The heat sources of the GaAs die are the 2 pHEMTs, modeled using the Curtice-cubic model, and the TaN bias resistors. The vias near the source terminals of the second stage pHEMT were modeled using a 2-port interface element, connected in parallel with the GaAs die. This is a good approximation of the embedded thermal subvolumes. The heat dissipation of the spiral inductors and other surface metallization was ignored.

Figure 3 shows the thermal image of the MMIC, with the temperature of the heat sink set to $35 \mathrm{C}$. Thermal camera emissivity calibrations and corrections were taken care of with a reference image of the MMIC, with no DC power. Also, during the correction process, the unevenness of the black ink, on certain areas of the MMIC, was taken into account. This unevenness of the black ink, reduces the emissivity of an area, leading to erroneous temperature readings. Hence even though the thermal image shows temperature readings above $50 \mathrm{C}$, the actual temperature readings of the various spots on the MMIC are shown in Table I. A purely thermal transient simulation, with no electrothermal feedback and a an electrothermal harmonic balance co-simulation with an input signal of $-10 \mathrm{dBm}$ at $10 \mathrm{GHz}$ at $T_{a m b}$ of $35 \mathrm{C}$, was performed. Table I compares the measured temperatures, of the 'pixel' elements at a heat sink temperature of $35 \mathrm{C}$, and the simulated steady-state temperatures. As seen in Figure 4, individual transistor finger temperatures, obtained from the electrothermal cosimulation, are higher than the 'pixel' element temperatures. The 'pixel' elements help calculate the average temperature over the resolution or 'pixel' size of the thermal camera. At these elevated temperatures, current reduction in the individual fingers, reduces their power dissipation. This low power dissipation at elevated temperatures, is accounted for in the electrothermal co-simulation, resulting in better simulated temperatures, as seen in Table I. Figure 5 shows the simulated steady-state output voltage of the MMIC with and without electrothermal coupling. If self-heating and mutual thermal interactions are ignored the output voltage is significantly overestimated, making them important considerations in the MMIC performance evaluation.

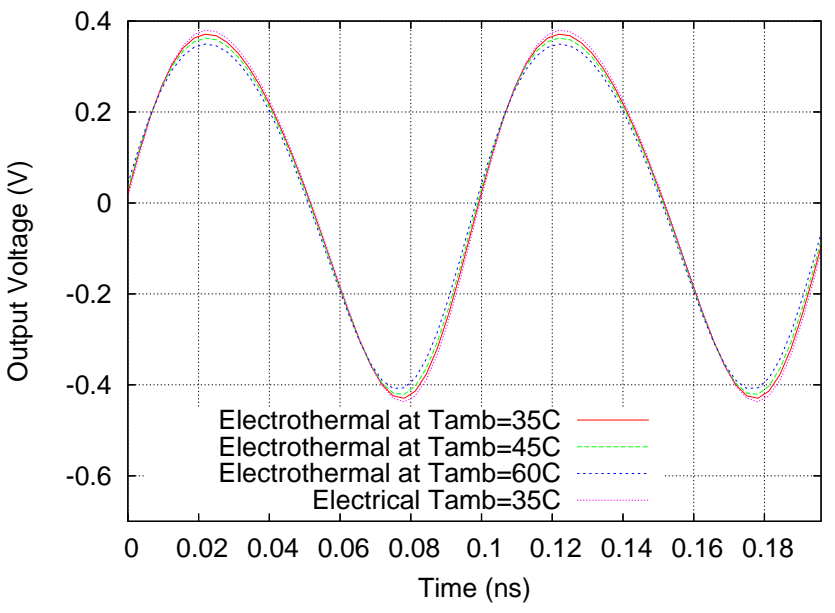

Fig. 5. Output voltage at elevated temperatures.

\section{CONCLUSION}

An electrothermal co-simulation of an X-band MMIC LNA has been demonstrated. The thermal impedance matrix model is a highly accurate model which describes thermal interactions, based on analytical solutions of the heat diffusion equation in thermal subvolumes. The concept of a universal error function has been demonstrated in the state variable based circuit simulator. The electrothermal steadystate simulations of the X-band MMIC under DC bias, have been validated with thermal images. This model will help predict effects of temperature rise due to self-heating and mutual interactions on the performance of MMICs.

\section{ACKNOWLEDGEMENT}

This material is based upon work supported in part by the Space and Naval Warfare Systems Center San Diego under grant number N66001-01-1-8921 through North Carolina State University as part of the DARPA NeoCAD Program.

\section{REFERENCES}

[1] C. E. Christoffersen, Global Modeling of Nonlinear Microwave circuits, Ph.D. Thesis, Department of Electrical and Computer Engineering, North Carolina State University.

[2] C. E. Christoffersen and M. B. Steer, "Implementation of the local reference node concept for spatially distributed circuits", Int. J. on $R F$ and Microwave Computer Aided Engineering, Vol. 9, No. 5, Sept. 1999, pp. 376-384.

[3] W. Batty, C. E. Christoffersen, A. J. Panks, S. David, C. M. Snowden, and M. B. Steer, "Electrothermal CAD of power devices and circuits with fully physical time-dependent compact thermal modeling of complex nonlinear 3-d systems", IEEE Trans. Components \& Packag. Technol., vol. 24, no. 4, pp. 566-590, December 2001.

[4] W. Veijola, L. Costa, and M. Valtonen, "An implementation of electrothermal component models in a general purpose circuit simulation programme," THERMINIC '97, Cannes, France, Sept. 21-23, 1997, pp. 96-100.

[5] A. R. Hefner and D. L. Blackburn, "An experimentally verified IGBT model implemented in the Saber circuit simulator", IEEE Transactions on Power Electronics", Vol. 9, No. 5, September 1994.

[6] V. Szekely, "THERMODEL:A tool for compact dynamic thermal model generation", Microelectron. J., vol. 29, pp. 257-267, 1998. 